\title{
Auditory temporal summation in infants and adults: Effects of stimulus bandwidth and masking noise
}

\author{
KATHLEEN M. BERG \\ University of Florida, Gainesville, Florida
}

\begin{abstract}
A visually reinforced operant procedure was employed to determine the behavioral thresholds of 6- to 7-month-old infants and adults for stimuli of various bandwidths and durations. Experiment 1 compared absolute thresholds for broadband and 1/3-octave filtered clicks and 300-msec noise bursts. For adult subjects, the difference in threshold for clicks and noise bursts was quite comparable in the two bandwidth conditions, but infants' click-noise threshold differences were significantly larger for broadband than for $1 / 3$-octave stimuli. In Experiment 2, 2-point thresholdduration functions were compared for $4-\mathrm{kHz}_{\mathrm{z}}$ tones and octave-band noise bursts presented in backgrounds of quiet and continuous noise. Infants' threshold-duration function for octave-band noise bursts was significantly steeper than the comparable adult function in quiet, but not in masking noise. These results suggest that young infants may have particular difficulty detecting lowintensity broadband sounds when durations are very short.
\end{abstract}

The relationship between the duration of an auditory stimulus and the intensity necessary for its detection has been the subject of numerous investigations during the past 4 to 5 decades, and still continues to be a topic of both theoretical and clinical interest. In general, auditory thresholds are found to decrease exponentially with increases in stimulus duration up to a maximum duration of approximately $200-300 \mathrm{msec}$. This decrease in threshold is thought to reflect the summation of neural activity within the auditory system over brief periods of time and is typically referred to as temporal summation or temporal integration. The time constant of integration varies with signal frequency; however, for midfrequency tones between durations of 10 and $100-200 \mathrm{msec}$, auditory thresholds closely approximate perfect integration of stimulus energy, decreasing at the rate of 8-10 dB per decade increase in stimulus duration (Gerken, Bhat, \& Hutchison-Clutter, 1990; Plomp \& Bouman, 1959; Watson \& Gengel, 1969).

Much of the interest in temporal summation stems from reports suggesting that alterations of the threshold-duration function may be systematically related to certain types of auditory dysfunction. For conditions in which the thresholds of normal-hearing adults decrease by approximately $10 \mathrm{~dB}$ for each decade increase in duration, shal-

This work was supported in part by a grant from the March of Dimes Foundation and by NIH Grant R01-DCO0017. I would like to thank Randy Limer, Susan Pastina, Tracy Marsh, and Kathy Shoucair for their assistance in testing subjects, and gratefully acknowledge the efforts of the many parents who generously contributed their own time and that of their infants to this project. Requests for reprints may be directed to the author at the Institute for Advanced Study of the Communication Processes, 63 Dauer Hall, University of Florida, Gainesville, FL 32611. low functions in the range of 3-6 dB per decade change in duration have been reported in subjects with hearing loss of cochlear origin (Florentine, Fastl, \& Buus, 1988; Wright, 1978; Wright \& Canella, 1969) and in normalhearing persons during recovery from auditory fatigue (Jerger, 1955; Mills, Gengel, Watson, \& Miller, 1970). The opposite effect, abnormally steep functions, has frequently been observed in instances of central auditory pathology, including lesions of brainstem pathways (Stephens, 1976) and the auditory cortex (Gersuni, Baru, Karaseva, \& Tonkonogii, 1971; Jerger, Lovering, \& Wertz, 1972; Stephens, 1976).

This apparent relation between the slope of temporal summation functions and integrity of the auditory system has led a number of investigators to ask whether temporal summation might also change during the course of normal development. Most studies have examined schoolage populations, with mixed results. In a comparison of normal and hearing-impaired children 8-12 years of age, Dempsey and Maxon (1982) determined thresholdduration functions for tones of several different frequencies and consistently found unusually steep functions, on the order of $15 \mathrm{~dB}$ per decade change in duration, in their normal-hearing subjects. Maxon and Hochberg (1982) reported similar steep functions in a sample of normal children 4-12 years of age. However, additional studies by Barry and Larson (1974), with 6- to 14-year-olds, and Olsen and Buckles (1979), with subjects ranging from 6 to 24 years of age, found no evidence that the temporal integration functions of normal children differed from those of adults.

More recently, two investigations of auditory temporal summation have been reported in young infants. In the first of these, Thorpe and Schneider (1987) found excep- 
tionally steep threshold-duration functions in a group of 6- to 7-month-old infants. They used a visually reinforced operant procedure to determine thresholds for $4 \mathrm{kHz}$ octave-band noise bursts, ranging from 50 to $517 \mathrm{msec}$ in duration, and found a threshold decrease in excess of $20 \mathrm{~dB}$ per decade change in duration over the range of linear integration. In contrast, Olsho and Marean (1988), using brief tone pips, found adult-infant differences in threshold to be nearly identical to those for long-duration stimuli. As a result, they concluded that temporal summation is mature by 3 months of age.

The pattern of inconsistent findings across studies suggests that nonsensory, perhaps attentional, factors may play an important role in developmental investigations of temporal integration. However, the discrepancy between results of the latter two experiments also raises the possibility that temporal summation functions of young infants may vary with the spectral content of test stimuli. Reported here are the results of two experiments that address this question. Both employed a visually reinforced head-turn paradigm in conjunction with a yes-no procedure to determine infants' sensitivity to stimuli of different durations. The first compared thresholds for broadband and $1 / 3$-octave clicks and noise bursts; the second examined 2-point threshold-duration functions for octaveband noise bursts and brief tones.

\section{EXPERIMENT 1}

\section{Method}

Subjects. The subjects were 22 normal, healthy infants tested when 26-29 weeks of age. They were assigned to either broadband or $1 / 3$-octave stimulus groups and administered each of two duration conditions in counterbalanced order. Within a session, testing was continued either until acceptable threshold estimates had been obtained for both conditions or until the infant became agitated or disinterested as determined by preset criteria. Sixteen infants ( 8 per group) completed the procedure successfully, requiring a mean of 1.3 sessions. Infants receiving broadband stimuli ( 5 males, 3 females) had a mean age of 27.1 weeks. Mean age of infants tested with $1 / 3$-octave stimuli ( 3 males, 5 females) was 27.5 weeks. Of the remaining 6 infants, 1 failed to meet the initjal criteria for conditioning and 5 failed to provide acceptable threshold estimates for both stimulus durations.

Sixteen young adult subjects were tested under the same conditions for comparison. One group, consisting of 4 males and 4 females, received broadband stimuli; a second group of 2 males and 6 females received the narrowband conditions. All were enrolled in introductory psychology courses and received class credit for their participation.

Apparatus and Procedure. Test stimuli were 300 -msec bursts of noise and brief clicks produced by single $0.1-\mathrm{msec}$ dc pulses. For broadband conditions, these stimuli were lowpass filtered at $10 \mathrm{kHz}$. Narrowband stimuli were generated by passing broadband clicks and noise bursts through an analog filter (24-dB/octave rolloff) set to pass a $1 / 3$-octave band centered at $4 \mathrm{kHz}$. Rise/decay time of the noise bursts was $3 \mathrm{msec}$. On a given trial, test stimuli were presented seven times with an onset-to-onset interval of $600 \mathrm{msec}$, resulting in a train approximately $4 \mathrm{sec}$ in duration. Timing of durations and intervals was accomplished by a laboratory computer, programmed to deliver the sequence of events for each trial and to vary intensity levels according to an up-down tracking procedure. All stimuli were presented in sound field via an Advent loudspeaker (Model 5002). Stimulus level, measured at the site of the infant's head by a Quest 215 sound level meter, did not vary more than $\pm 2 \mathrm{~dB}$ within the range of possible head positions.

Testing was conducted within a single-walled IAC soundattenuated chamber while the infant was seated on the parent's lap, facing an observer/entertainer across a small table. The loudspeaker and three smoked-Plexiglas boxes, each containing a batteryoperated toy, were situated $90^{\circ}$ to the infant's left at a distance of $0.8 \mathrm{~m}$. The entertainer displayed colorful toys to engage the infant's attention and pressed a foot-operated pedal to initiate trials when the infant was judged to be attentive and in midline orientation. During testing, both the parent and entertainer received masking noise through headphones to eliminate possible sources of bias.

Each trial consisted of a 4-sec observation interval marked by a signal delivered via headphones to a second observer outside the chamber. During this interval, the stimulus train was presented with a probability of .5 . Head turns to the left within the 4-sec observation interval were considered positive responses and were registered, along with failures to turn, on a response box by the observer seated outside the booth. The order of signal and nonsignal trials was random, with the restriction that no more than three trials of either type be presented consecutively. Head turns on signal trials were reinforced by immediate activation of the toy and illumination of colored lights within one of the Plexiglas boxes. The duration of the reinforcer was $3 \mathrm{sec}$.

Prior to threshold determination, each infant was required to meet a conditioning criterion, with signal duration equal to $300 \mathrm{msec}$ and signal level fixed at $60 \mathrm{~dB}$ SPL. The criterion was set at five consecutive correct responses for the initial session and two consecutive correct responses for subsequent sessions. Two consecutive correct responses at conditioning level were also required following within-session changes in stimulus duration. Conditioning level for click stimuli was $80 \mathrm{~dB}$ "peak equivalent" SPL (peSPL).

Once the conditioning criterion had been met, thresholds were determined using an adaptive up-down tracking procedure. Signal level was initially set $20 \mathrm{~dB}$ above adult threshold for the same condition and varied in 8-dB steps until the first up-down reversal. Step size was then reduced to $4 \mathrm{~dB}$, and tracking continued, with two consecutive correct responses required for a 4-dB decrease in level and a single failure to respond resulting in a 4-dB increase in level. This procedure converges on the signal intensity level yielding approximately $71 \%$ correct responses (Levitt, 1971). After the first reversal, suprathreshold probe trials with the signal set at conditioning level were presented every sixth trial to assess the infant's degree of interest. Tracking continued until the fourth up-down reversal; threshold estimates were computed as the mean stimulus level over the last two reversals. If false alarms occurred on more than one third of the nonsignal trials or if the infant failed to respond on more than two probe trials, the threshold estimate was considered unacceptable and the stimulus condition was repeated. Within a given session, testing continued until the infant became fussy or inattentive as indicated by failure to respond on two consecutive probe trials. Average length of sessions was approximately $20 \mathrm{~min}$.

Procedures for the adult subjects were intended to duplicate the critical aspects of the infant test situation as closely as possible. Adults were provided with a response box and instructed to press a button whenever they heard the stimuli. Buttonpresses during the 4-sec observation interval were considered positive responses; failures to press during this interval were interpreted as negative responses. Intertrial intervals, measured from the end of one trial to the beginning of the next, varied randomly from 1 to $15 \mathrm{sec}$ in duration, so that time of signal presentations was uncertain. Feed- 
back for correct detections was provided by the brief activation of lights within one of the Plexiglas boxes. All other procedures were identical to those described for infants.

\section{Results and Discussion}

Threshold estimates for clicks and 300-msec noise bursts are shown for both broadband and $1 / 3$-octave stimulus groups in Figure 1. Error bars represent \pm 1 standard deviation from the mean. Thresholds for noise stimuli are given in decibels (SPL), and those for clicks are expressed as decibels (peSPL). In general, both adult and infant threshold estimates were in good agreement with existing data. For 300-msec noise stimuli, infant thresholds averaged $15 \mathrm{~dB}$ above those of adults, well within the range of infant-adult differences reported in similar studies of auditory sensitivity during infancy (Olsho, Koch, Carter, Halpin, \& Spetner, 1988; Trehub, Schneider, \& Endman, 1980).

For adult subjects, the difference in threshold for clicks and noise bursts was quite comparable in the two bandwidth conditions; mean click thresholds were 22.5 and $25 \mathrm{~dB}$ above noise-burst thresholds for broadband and filtered conditions, respectively. This was not the case for infant subjects, whose click-noise threshold differences were substantially larger for broadband $(39 \mathrm{~dB})$ than for narrowband $(27 \mathrm{~dB})$ stimuli. An analysis of variance confirmed that the three-way interaction of age, stimulus bandwidth, and duration was highly significant $[F(1,28)$ $=29.26, p<.001]$.

There were no reliable differences among conditions in number of trials required to reach threshold, but overall false alarm rate was significantly higher for infants (mean $=.17)$ than for adults (mean $=.02)[F(1,28)=$ 28.97, $p<.001]$. Percentage of false alarms also showed

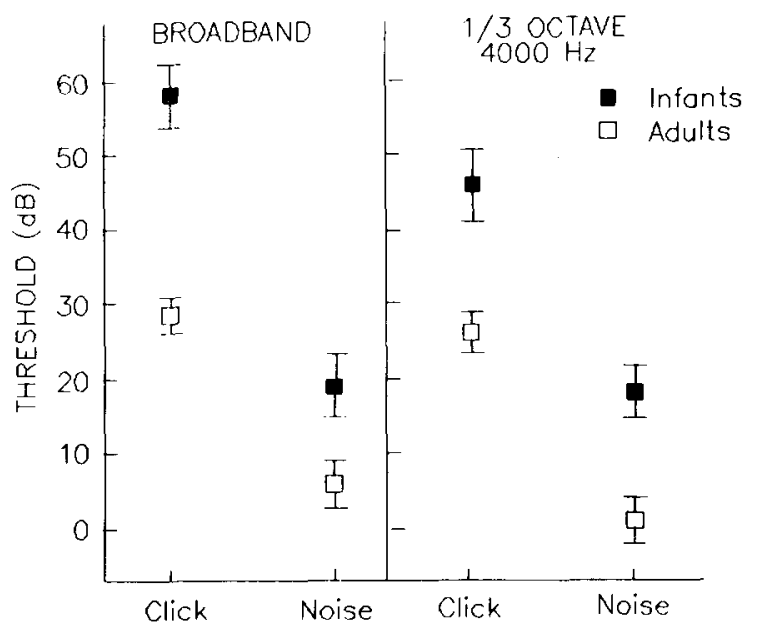

Figure 1. Infant and adult thresholds for broadband and $1 / 3-$ octave filtered clicks and 300-msec noise bursts. Center frequency of $1 / 3-$ octave stimuli was $\mathbf{4} \mathbf{k H z}$. Noise thresholds are given in decibels (SPL); click thresholds are given in decibels (peSPL). Error bars represent \pm 1 standard deviation from the mean. a significant bandwidth $\times$ duration interaction $[F(1,28)=$ $5.61, p<.05]$, but did not differ for click and noise stimuli when broadband and $1 / 3$-octave groups were analyzed separately. Thus, it is unlikely that the observed differences in threshold were secondary to differences in false-alarm rate.

These results confirm the discrepant findings of previous studies and suggest that the slope of threshold-duration functions may be considerably steeper for broadband than for narrowband stimuli in 6- to 7-month-old infants. Although a number of authors have speculated on mechanisms that might underlie adult-infant differences in sensitivity to longer duration sounds, none of these appears likely to account for differences in temporal summation. One possible explanation, suggested by the consistently higher thresholds of younger subjects, is that infants may have been responding on the basis of loudness of the test stimuli. Results of studies investigating the relation between loudness and duration are quite variable, but, as a general rule, neither stimulus bandwidth nor level appear to have consistent effects on temporal summation of loudness in adult subjects (Scharf, 1978). These negative findings suggest that loudness of the stimuli was probably not a critical factor. Clinically, unusually steep temporal integration functions have been associated with lesions of central auditory pathways (Gersuni et al., 1971; Stephens, 1976). However, in these instances the steep pattern is seen for tones as well as for broadband noise and, therefore, probably involves different mechanisms. In the absence of known auditory dysfunction, exceptionally steep slopes are frequently indicative of inattentiveness or lack of cooperation on the part of the subject (Wright, 1978). While it is always difficult to completely rule out the contribution of such performance or task variables, the fact that infants' threshold-duration functions were not steeper than adults' for $1 / 3$-octave stimuli argues against this interpretation. A second experiment was therefore undertaken to replicate these findings and to clarify the nature of the processes involved.

\section{EXPERIMENT 2}

One obvious question raised by the results of Experiment 1 is whether infants' difficulty in detecting broadband, short-duration sounds represents an effect of stimulus bandwidth per se or whether it reflects an alteration in the function relating absolute threshold to frequency. Although the audibility curves of 6-month-olds and adults are quite similar for long-duration stimuli (Olsho et al., 1988), there are no data to confirm that this is also the case when stimuli are very brief. A second question is whether the exceptionally steep temporal integration pattern seen in young infants at absolute threshold would also be observed at higher stimulus levels. These questions were addressed in Experiment 2 by comparing the 2-point threshold-duration functions of infants and adults in backgrounds of quiet and masking noise. Stimuli were $4-\mathrm{kHz}$ 
tones and octave-band noise bursts, selected to limit stimulus energy to a relatively narrow region of the frequency spectrum.

\section{Method}

Subjects and Procedure. Twenty normal, healthy infants received octave-band noise bursts as test stimuli. Upon arrival at the laboratory, they were randomly assigned to either absolute or masked threshold groups and administered each of two duration conditions in counterbalanced order. Sixteen infants ( 8 per group) completed both conditions successfully, requiring a mean of 1.1 sessions. Infants tested in quiet (4 males, 4 females) had a mean age of 27.1 weeks (range $=25-28$ weeks). Mean age of infants tested in the noise background ( 6 males, 2 females) was 27.8 weeks (range = 26-30 weeks). Of the remaining 4 infants, 1 failed to meet the initial criteria for conditioning and 3 failed to provide acceptable threshold estimates for both duration conditions. Procedures used to determine thresholds were identical to those described for Experiment 1 .

Several methodological changes were introduced for the subjects receiving tone stimuli. One of these was the use of lightweight headphones (Sony MDR-70II) for stimulus presentation. Two others, reduction of the minimum step size from 4 to $2 \mathrm{~dB}$ and an increase in number of reversals from four to five, were intended to increase the accuracy of threshold tracking. These changes resulted in a slight increase in subject loss; 26 infants were tested in order to obtain a sample of 16 , who required a mean of 1.4 sessions to provide acceptable threshold estimates for the two duration conditions. Both absolute- and masked-threshold groups consisted of 2 males and 6 females. Infants tested in quiet had a mean age of 28 weeks (range = 27-31 weeks); infants tested in the background of noise had a mean age of 27.8 weeks (range $=26-30$ weeks). Of the 10 infants replaced, 1 would not tolerate the headphones, 2 failed to meet the criteria for conditioning, and 7 lost interest in the reinforcers before acceptable threshold estimates could be obtained for both duration conditions.

Sixteen young adults recruited from the same population as those serving in Experiment 1 were tested in both quiet and noise background conditions during a single 30 -min session. Eight subjects ( 4 males, 4 females) were tested with octave-band noise bursts, and the remaining subjects ( 2 males, 6 females) received tones.

Octave-band noise bursts centered at $4 \mathrm{kHz}$ were produced by passing white noise through an analog filter with a $24-\mathrm{dB} /$ octave roll-off. They were presented in sound field, with a rise/decay time of $3 \mathrm{msec}$ and durations of 10 and $300 \mathrm{msec}$, as measured at halfpower points. The $4-\mathrm{kHz}$ tones were presented to the left ear via the headphone, with rise/decay times of $3 \mathrm{msec}$ and durations of 10 and $100 \mathrm{msec}$. As in Experiment 1, the stimuli were repeated in 4-sec trains. For subjects in masked-threshold groups, test stimuli were presented over a background of continuous noise, generated by an independent source and bandpass filtered from $2-6 \mathrm{kHz}$. Noise intensity level was $46 \mathrm{~dB}$ SPL (spectrum level $=10 \mathrm{~dB} / \mathrm{Hz}$ ). Approximately half of the infants receiving tone stimuli were tested by a single experimenter who served as entertainer and also made judgments on head turns. In these instances, the observation-interval signal was added to the masking noise in the experimenter's headphones.

For tone groups, tracking began with signal level set at either

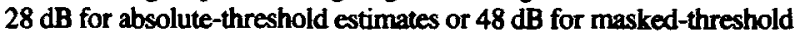
estimates. Step size, initially fixed at $8 \mathrm{~dB}$, was reduced by half on subsequent reversals until the minimum step size of $2 \mathrm{~dB}$ had been reached. Stimulus level then varied in $2-\mathrm{dB}$ steps until the fifth up-down reversal, when tracking was terminated. As in Experiment 1 , thresholds were computed as the mean signal level over the last two reversals.

\section{Results and Discussion}

Mean infant and adult thresholds for octave-band noise bursts are shown in Figure 2; comparable threshold estimates for tone stimuli are illustrated in Figure 3. Absolute and masked-threshold estimates are presented in the left and right panels of each figure, respectively. Despite the numerous differences in procedure, thresholds for both age groups appear to be consistent with results of previous studies. In quiet, adult-infant differences were nearly identical for octave-band noise and tone groups: mean infant thresholds exceeded adult estimates by $13 \mathrm{~dB}$ for $300-$ msec noise bursts and by $14 \mathrm{~dB}$ for 100 -msec tones. In

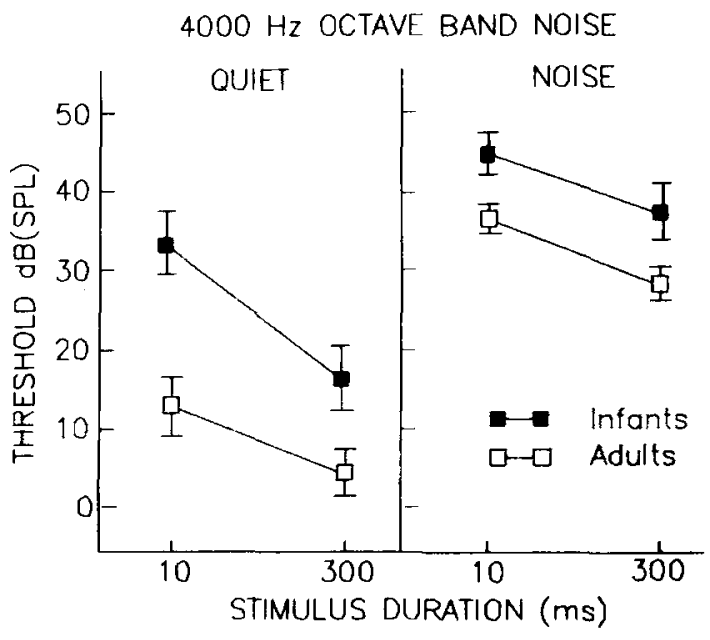

Figure 2. Infant and adult thresholds for $4-k H z$ octave-band noise bursts, 10 and 300 msec in duration, presented in backgrounds of quiet and 10-dB/Hz masking noise. Error bars represent \pm 1 standard deviation from the mean.

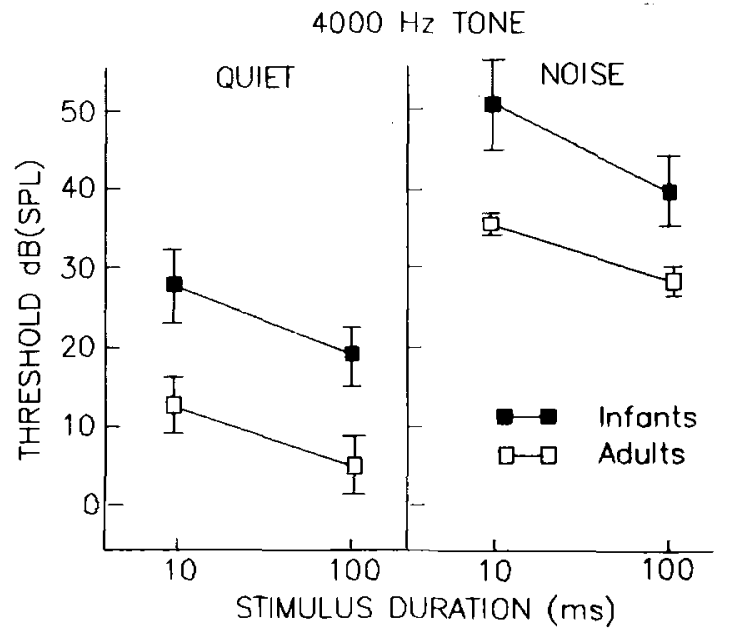

Figure 3. Infant and adult thresholds for $4-\mathrm{kHz}$ tones, 10 and $100 \mathrm{msec}$ in duration, presented in backgrounds of quiet and 10$\mathrm{dB} / \mathrm{Hz}$ masking noise. Error bars represent \pm 1 standard deviation from the mean. 
background noise, thresholds for longer duration stimuli were increased by approximately $21 \mathrm{~dB}$ in infants and by $24 \mathrm{~dB}$ in adults, in good agreement with Nozza and Wilson's (1984) report that monaural masking noise produces a similar elevation of threshold in adult and infant subjects. Adult signal-to-noise ratios for both tones and noise bursts were slightly smaller than values reported in the literature (Zwislocki, 1978), possibly because of the use of stimulus trains rather than single presentations.

For adult subjects, the 2-point threshold-duration functions showed little variation as a function of spectral content or as a function of background condition. Thresholds for 10- and 300-msec octave-band noise bursts differed by $8.5 \mathrm{~dB}$ in quiet and by $7.7 \mathrm{~dB}$ in masking noise. For 10 - and 100 -msec tone stimuli, threshold differences were $7.6 \mathrm{~dB}$ in quiet and $7.0 \mathrm{~dB}$ in noise.

In contrast, infant threshold-duration functions were affected by both stimulus bandwidth and background conditions. For octave-band noise bursts presented in quiet, thresholds decreased by nearly $16 \mathrm{~dB}$ as duration increased from 10 to $300 \mathrm{msec}$. When continuous masking noise was present in the background, this difference was reduced to $6.5 \mathrm{~dB}$. The pattern was reversed for tone stimuli; mean thresholds for 10 - and 100 -msec durations differed by $11 \mathrm{~dB}$ in masking noise, but by only $8.5 \mathrm{~dB}$ in quiet. An analysis of variance indicated that this interaction was significant [stimulus $\times$ background $\times$ duration, $F(1,28)=12.13, p<.005$ ]. Separate analyses of tone and noise groups revealed that integration functions for noise bursts in quiet and noise backgrounds were significantly different [background $\times$ duration, $F(1,14)=$ $15.98, p<.005$ ], but background effects on functions for tones failed to reach significance.

Further analyses including both infant and adult subjects confirmed that for octave-band noise bursts presented in quiet, infants' temporal summation functions were significantly steeper than were adults' [age $\times$ duration, $F(1,14)=16.73, p<.005$ ], but not for tones in quiet $(F<1)$. In a comparison of adult and infant thresholds for tones presented in noise, it was determined that group variances departed significantly from homogeneity $[F(1,14)=7.89, p<.01]$. When procedures not requiring equal group variances were employed (Dixon, 1988), the difference in slopes of temporal summation functions for the two age groups remained marginally significant $[F(1,8)=5.65, p<.05]$. The slopes of adult and infant functions for octave-band noise bursts in masking noise did not differ $(F<1)$.

There were no significant differences between durations in number of trials required to estimate threshold, but subjects in the tone groups required significantly more trials than did those tested with octave-band noise [ 33.5 vs. $25.8 ; F(1,62)=43.00, p<.001]$. Percentage of false alarms did not differ among conditions, although overall false-alarm rate was significantly higher for infants than for adults in both noise and tone groups [for octave-band noise, .21 vs. $.02, F(1,28)=94.72, p<.001$; for tones, .18 vs. $.06, F(1,28)=20.28, p<.001]$.

\section{GENERAL DISCUSSION}

Considered together, results of these two experiments suggest that both the spectral characteristics of the stimulus and the background conditions are important determinants of temporal summation in young infants. When stimuli were presented in a quiet background, infants' temporal summation functions for tones and $1 / 3$-octave noise bursts were nearly identical in slope to those of adults. Thresholds for 4-kHz tones decreased at the rate of $8.5 \mathrm{~dB}$ per decade in infants and $7.6 \mathrm{~dB}$ per decade in adults, in good agreement with the slope of 7-8 $\mathrm{dB}$ per decade change in duration reported for similar stimuli in the adult literature (Gerken et al., 1990). In contrast, infant threshold-duration functions for broadband signals were significantly steeper than were comparable adult functions. This difference in slope was seen for tones and noise contained within a 1-octave bandwidth, suggesting that the steep slope of infants' broadband functions is not solely attributable to variations in sensitivity to brief stimuli across the frequency spectrum.

When low-level masking noise was introduced in the background, the effect of stimulus bandwidth on infants' threshold-duration functions was reversed; the infant function for tones was significantly steeper than the adult function, whereas the infant function for octave-band noise was not steeper than the adult function. These divergent changes in slope for tones and noise bursts were unexpected, and they suggest that more than one factor may have contributed to the results. In view of the welldocumented relationship between unusually steep temporal integration functions and motivational or attentional variables, it seems likely that infants' steeper function for tones in noise reflects the operation of cognitive, rather than sensory, factors. A study reported by Tucker, Williams, and Jeffress (1968) in adult subjects provides some support for this conclusion. These investigators examined detectability of tones in masking noise and found poorer performance at short durations in naive, but not in wellpracticed, observers - a result they attributed to increased difficulty of the detection task when stimuli are very brief. Uncertainty about the temporal parameters of the signal has been cited as a possible factor contributing to this increased task difficulty. Although the use of stimulus trains in the present experiments would be expected to minimize uncertainty, young infants may have been less efficient than adults in utilizing the information available in multiple stimulus presentations. Duration-specific differences in task difficulty may have also influenced thresholds for noise bursts, but since infants' temporal integration slopes for noise bursts in background noise were significantly flatter than those in quiet, such influences were presumably minimal.

If infants' elevated thresholds for brief tones in noise can be attributed to nonauditory factors, the remaining question to be answered is why their threshold-duration functions for broadband stimuli should be steeper than adults' in quiet, but not in masking noise. There appear 
to be at least three possible interpretations of this finding and very little evidence to assist in selecting among them. One explanation would attribute age differences in temporal summation to developmental differences in the neural representation of stimulus intensity. Neurophysiological studies have indicated that the slope of threshold-duration functions probably derives from the coding of intensity and can be predicted from the spike rate-intensity functions of peripheral auditory neurons (Fay \& Coombs, 1983). Furthermore, the slope and dynamic range of peripheral rate-intensity functions have been reported to change during the course of normal auditory development (Carlier \& Pujol, 1978; Romand, 1983). The few available studies of intensity discrimination in human infants suggest that under optimal conditions their sensitivity to increments in intensity is not very different from that of adults (e.g., Sinnott \& Aslin, 1985). However, the development of intensity discrimination has not been systematically examined as a function of either stimulus level or bandwidth, and the possibility that intensity coding is markedly different in 6-month-olds and adults cannot be ruled out.

A second possible interpretation would ascribe age differences in temporal summation to more central selective-attention mechanisms. According to traditional models of the auditory system, stimulus energy is integrated within "critical bands" slightly smaller than $1 / 3$ octave in bandwidth, which behave as independent frequency channels (Garner, 1947). When stimuli are noise bursts or other complex sounds exceeding the width of a single critical-band channel, subjects are able to adjust their listening strategies and combine information across as many channels as necessary to achieve optimal performance (Buus, Schorer, Florentine, \& Zwicker, 1986; Green, 1960; Swets, 1963). Thus, it may be that infants demonstrate adult-like temporal integration within channels but are less efficient than adults in monitoring more than one channel or combining information across channels when stimuli are very brief. Such models are also able to account for the equivalence of adult and infant performance in masking noise: since the signal-to-noise ratio remains constant for listening bands less than or equal to the bandwidth of the signal, an inability to integrate across the frequency spectrum would not be a disadvantage.

A third alternative proposes that differences in the threshold-duration functions of infants and adults are due to the immaturity of a spectral integration process specific to stimulus onsets. This mechanism is suggested by results of several studies demonstrating that variations in the level of masking noise may produce nonlinear effects on performance that are apparent only when stimuli are very brief. Nonlinear masking effects have been reported in a number of paradigms, including detection of tone and noise bursts (Carlyon \& Moore, 1986; Stephens, 1973), detection of tone glides (Collins \& Cullen, 1984), intensity discrimination (Carlyon \& Moore, 1984), and "overshoot," the increase in detectability of a brief signal as it is delayed relative to onset of the masker (Bacon, 1990).
In most instances, investigators have attributed these nonlinear effects to two distinct populations of auditory nerve fibers, differing in threshold and onset response (Rhode \& Smith, 1985). While there is as yet no evidence to link a specific low-threshold fiber population with spectral integration of brief signals, van den Brink and Houtgast's (1990) recent report of enhanced efficiency in acrossfrequency integration of brief transients suggests that such a duration-specific process may exist. Whether it also matures more slowly than mechanisms underlying spectral integration of longer stimuli remains to be determined.

\section{REFERENCES}

Bacon, S. P. (1990). Effect of masker level on overshoot. Joumal of the Acoustical Society of America, 88, 698-702.

BARRY, S. J., \& LARSON, V. D. (1974). Brief-tone audiometry with normal and deaf school-age children. Journal of Speech \& Hearing Disorders, 39, 457-464.

Buus, S., Schorer, E., Florentine, M., Zwicker, E. (1988). Decision rules in detection of simple and complex tones. Joumal of the Acoustical Society of America, 80, 1646-1657.

Caruer, E., Pujol, R. (1978). Role of inner and outer hair cells in coding sound intensity: An ontogenetic approach. Brain Research, 147, 174-176.

CARLYON, R. P., MOORE, B. C. J. (1984). Intensity discrimination: A severe departure from Weber's law. Journal of the Acoustical Society of America, 76, 1369-1376.

CARlyon, R. P., \& MoORE, B. C. J. (1986). Detection of tones in noise and the "severe departure" from Weber's law. Joumal of the Acoustical Society of America, 79, 461-464.

Collins, M. J., CulleN, J. K., JR. (1984). Effects of background noise level on detection of tone glides. Jourmal of the Acoustical Society of America, 76, 1696-1698.

DempSEY, J. J., \& MAXON, A. B. (1982). Temporal integration functions in hearing-impaired children. Ear \& Hearing, 3, 271-273.

Dixon, W. J. (1988). BMDP statistical sofiware manual. Berkeley: University of California Press.

FAY, R. R., COOMBS, S. (1983). Neural mechanisms in sound detection and temporal summation. Hearing Research, 10, 69-92.

Florentine, M., Fastl, H., \& BuUs, S. (1988). Temporal integration in normal hearing, cochlear impairment, and impairment simulated by masking. Journal of the Acoustical Society of America, 84, 195-203.

GARNER, W. R. (1947). The effect of frequency spectrum on temporal integration of energy in the ear. Journal of the Acoustical Society of America, 19, 808-815.

Gerken, G. M., Bhat, V. K. H., \& Hutchison-Clutter, M. (1990). Auditory temporal integration and the power function model. Journal of the Acoustical Society of America, 88, 767-778.

Gersuni, G., BArU, A., Karaseva, T., Tonkonogi, I. (1971). Effects of temporal lobe lesions on perception of sounds of short duration. In G. V. Gersuni (Ed.), Sensory processes at the neuronal and behavioral levels (pp. 287-300). New York: Academic Press.

GREEN, D. M. (1960). Auditory detection of a noise signal. Journal of the Acoustical Society of America, 32, 121-131.

JERGER, J. (1955). Influence of stimulus duration on the pure-tone threshold during recovery from auditory fatigue. Joumal of the Acoustical Society of America, 27, 121-124.

Jerger, J., Lovering, L., Wertz, M. (1972). Auditory disorder following bilateral temporal lobe insult: Report of a case. Joumal of Speech \& Hearing Disorders, 37, 523-535.

LEVITT, H. (1971). Transformed up-down methods in psychoacoustics. Joumal of the Acoustical Society of America, 49, 467-477.

Maxon, A. B., HochBeRg, I. (1982). Development of psychoacoustic behavior: Sensitivity and discrimination. Ear \& Hearing, 3, 301-308.

Mills, J. H., Gengel, R. W., Watson, C. S., \& Miller, J. D. (1970). Temporary changes of the auditory system due to exposure to noise 
for one or two days. Journal of the Acoustical Society of America 48, 524-530.

NozzA, R. J., \& WILSON, W. R. (1984). Masked and unmasked puretone thresholds of infants and adults: Development of auditory frequency selectivity and sensitivity. Journal of Speech \& Hearing Research, 27, 613-622.

OLSEN, C. C., \& BUCKLES, K. M. (1979). The effect of age in brieftone audiometry. Journal of Auditory Research, 19, 117-122.

Olsho, L. W., Koch, E. G., Carter, E. A., Halpin, C. F., \& SpetNER, N. B. (1988). Pure-tone sensitivity of human infants. Journal of the Acoustical Society of America, 84, 1316-1324.

Olsho, L. W., Marean, G. C. (1988). Infant thresholds for short duration tone bursts. Journal of the Acoustical Society of America, 84, S144.

Plomp, R., \& Bouman, M. A. (1959). Relation between hearing threshold and duration for tone pulses. Joumal of the Acoustical Society of America, 31, 749-758.

Rhode, W. S., \& SMITH, P. H. (1985). Characteristics of tone-pip response patterns in relationship to spontaneous rate in cat auditory nerve fibers. Hearing Research, 18, 159-168.

Romand, R. (1983). Development of the cochlea. In R. Romand (Ed.), Development of auditory and vestibular systems (pp. 47-88). New York: Academic Press.

Scharf, B. (1978). Loudness. In E. C. Carterette \& M. P. Friedman (Eds.), Handbook of perception: Vol. IV. Hearing (pp. 187-242). New York: Academic Press.

SinNotT, J. M., \& AsLin, R. N. (1985). Frequency and intensity discrimination in human infants and adults. Journal of the Acoustical Society of America, 78, 1986-1992.

STEPHENS, S. D. G. (1976). Auditory temporal summation in patients with central nervous system lesions. In S. D. G. Stephens (Ed.), Disorders of auditory function II (pp. 231-241). New York: Academic Press.
Stephens, S. D. G. (1973). Auditory temporal integration as a function of intensity. Journal of Sound \& Vibration, 30, 109-126.

SWETS, J. A. (1963). Central factors in auditory frequency selectivity. Psychological Bulletin, 60, 429-440.

ThORPE, L. A., SChNeIder, B. A. (1987, April). Temporal integration in infant audition. Paper presented at the meeting of the Society for Research in Child Development, Baltimore, MD.

Trehub, S. E., Schneider, B. A., Endman, M. (1980). Developmental changes in infants' sensitivity to octave-band noises. Journal of Experimental Child Psychology, 29, 282-293.

Tucker, A., Williams, P. I., Jeffress, L. A. (1968). Effect of signal duration on detection for gated and for continuous noise. Joumal of the Acoustical Society of America, 44, 813-816.

VAN DEN BrinK, W. A. C., \& Houtgast, T. (1990). Efficient acrossfrequency integration in short-signal detection. Journal of the Acoustical Society of America, 87, 284-291.

Watson, C. S., Gengel, R. W. (1969). Signal duration and signal frequency in relation to auditory sensitivity. Journal of the Acoustical Society of America, 46, 989-997.

WRIGHT, H. N. (1978). Brief tone audiometry. In J. Katz (Ed.), Handbook of clinical audiology (pp. 218-232). Baltimore, MD.: Williams \& Wilkins.

Wright, H. N., \& CanelLa, F. (1969). Differential effect of conductive hearing loss on the threshold-duration function. Journal of Speech \& Hearing Research, 12, 607-615.

ZWISLOCKI, J. J. (1978). Masking: Experimental and theoretical aspects of simultaneous, forward, backward, and central masking. In E. C. Carterette \& M. P. Friedman (Eds.), Handbook of perception: Vol. IV. Hearing (pp. 283-336). New York: Academic Press.

(Manuscript received October 9, 1990; revision accepted for publication May 20, 1991.) 